\title{
RISCO CLIMÁTICO DE OCORRÊNCIA DE DOENÇAS FÚNGICAS NA VIDEIRA 'NIAGARA ROSADA' NA REGIÃO DO POLO TURÍSTICO DO CIRCUITO DAS FRUTAS DO ESTADO DE SÃO PAULO (1)
}

\author{
LUDMILA BARDIN $\left(2^{*}\right)$; MÁRIO JOSÉ PEDRO JÚNIOR $\left({ }^{3,4}\right)$; JENER FERNANDO LEITE DE MORAES $\left({ }^{3}\right)$
}

\begin{abstract}
RESUMO
O Polo Turístico do Circuito das Frutas do Estado de São Paulo congrega municípios produtores de uva comum de mesa, principalmente a cultivar "Niagara Rosada". Observa-se que no processo produtivo ocorre severa influência de doenças fúngicas foliares e seu controle chega a 30\% do custo de produção. Nesse contexto, o objetivo foi determinar o risco climático $(R C)$ de ocorrência das doenças fúngicas na 'Niagara Rosada' por meio da análise de séries históricas de dados pluviométricos diários para estimativa do número de pulverizações necessárias $\left(N P_{\text {chuva }}\right)$. $\mathrm{O} R \mathrm{C}$, assim como o $N P_{\text {chuva' }}$, foram calculados em cada época de poda, e para os diferentes municípios da região. A regionalização das áreas em função da necessidade de pulverização para controle de doenças fúngicas determinada pela ocorrência de chuvas, foi feita por meio de sistemas de informações geográficas (SIG) utilizando os valores médios de $R C$ estimados, por equações de regressão linear, obtidas em função da altitude. Nas podas efetuadas em setembro, o RC foi mais elevado que nas de julho, sendo os valores mais elevados superiores a $70 \%$ e os menores, inferiores a $20 \%$ respectivamente.
\end{abstract}

Palavras-chave: viticultura, precipitação pluvial, época de colheita, controle fitossanitário.

\author{
ABSTRACT \\ CLIMATIC RISK INDEX OF FUNGAL DISEASES OCCURRENCE IN ‘NIAGARA ROSADA’ GRAPEVINE \\ GROWING IN THE POLO TURÍSTICO DO CIRCUITO DAS FRUTAS, SÃO PAULO STATE, BRAZIL
}

The "Polo Turístico do Circuito das Frutas" region congregates counties that produce "Niagara Rosada" grapevine. The grapevines suffer severe influence of fungal diseases and their control reach up to $30 \%$ of the production cost. Aiming to determine the climatic risk (CR) for 'Niagara Rosada' grapevine fungal diseases occurrence, a rainfall data bank was used to estimate the necessary number of sprays to control the main fungal diseases. The CR, as well as the necessary number of sprays, was calculated for the different probable harvest dates, for each pruning date at the different counties. The characterization of the different growing areas considering the higher need of fungicide sprays to control fungal diseases as a function of rainfall occurrence was done by means of GIS, using estimated values of CR through regression equation based on altitude. The September pruning dates presented higher CR values than July, being the highest values over $70 \%$ and the lowest under $20 \%$, respectively.

Key words: viticulture, rainfall, harvesting date, phytosanitary control.

(1) Recebido para publicação em 6 de janeiro de 2009 e aceito em 23 de março de 2010.

$\left({ }^{2}\right)$ Aluna de Pós-Graduação em Agricultura Tropical e Subtropical, área de Gestão de Recursos Agroambientais, Instituto Agronômico (IAC), Caixa Postal 28, 13001-970 Campinas (SP). Bolsista FAPESP. E-mail: ludmila_bardin@yahoo.com.br ( ${ }^{*}$ ) Autora correspondente.

(3) Instituto Agronômico (IAC), Campinas (SP).

$\left({ }^{4}\right)$ Bolsista CNPq. 


\section{INTRODUÇÃO}

A Região Leste do Estado de São Paulo formada pelos municípios de Indaiatuba, Itupeva, Itatiba, Jarinu, Jundiaí, Louveira, Valinhos, Vinhedo e Atibaia é um polo de referência em fruticultura no Estado de São Paulo. Por ser uma região de perfil agrícola, a produção de frutas tem sido a base econômica para muitos produtores, incentivando o turismo rural, pois os cultivos são predominantemente em pequenas propriedades, favorecendo a visitação do turista e possibilitando a geração de empregos.

Dentre as frutíferas que caracterizam o processo produtivo da região destaca-se a videira, sendo a uva 'Niagara Rosada' uma das culturas de maior importância para a região. As condições climáticas regionais são favoráveis à sua produção, mas também à ocorrência de doenças fúngicas, principalmente antracnose, míldio e mancha-das-folhas, sendo estas doenças, motivo de perdas na produção. O controle, realizado com base em aplicação de defensivos agrícolas, onera o custo de produção e impacta o meio ambiente. Nas áreas onde as condições climáticas são favoráveis ao desenvolvimento de patógenos, seu controle pode atingir até $30 \%$ do custo de produção (SÔNEGO et al., 2006).

Visando racionalizar o uso de fungicidas, vários métodos de previsão de época de aplicação foram desenvolvidos com base na inter-relação clima-patógeno-hospedeiro, para diminuir o custo da produção e o impacto ambiental. A maioria deles indica a oportunidade de pulverização em função da ocorrência de chuvas. BLEICHER (1991) avaliou um sistema pluviométrico para previsão da podridão amarga da macieira e Pezzopane (1998) para o controle da manchapreta do amendoim.

No caso da videira 'Niagara Rosada' a validade do uso de totais pluviométricos para previsão de épocas de aplicação de fungicida visando ao controle de antracnose, míldio e mancha-das-folhas, em videira 'Niagara Rosada', foi testada por PEDRO JÚNIOR et al. (1999) na região de Jundiaí (SP). Os resultados mostraram que a aplicação de fungicida após ocorrência de $20 \mathrm{~mm}$ de chuva controlou satisfatoriamente as doenças fúngicas na videira, principalmente, a antracnose nos cachos, folhas e ramos, permitindo a redução de aproximadamente $40 \%$ do número total de pulverizações necessárias.

O estudo de séries históricas de ocorrência de chuva para estimativa da necessidade de pulverização e avaliação do risco climático pela combinação de diferentes técnicas agrometeorológicas e modelos de previsão da ocorrência de doenças em culturas, permite a geração de mapas que melhoram a visualização e distribuição das áreas de risco à ocorrência das doenças (Lozada Garcia, 2005). Neste contexto, Booth et al. (2000) determinou áreas de escape de ocorrência da requeima em eucalipto, na África, Austrália, América Latina e sudoeste da Ásia. LozAda Garcia (2005); ZeUNER e KLEINHENZ (2007) avaliaram o risco climático de ocorrência da requeima da batata na Venezuela e na Alemanha respectivamente. Segundo os autores, SIG e modelos de previsão podem facilitar o controle da doença e pode-se alcançar uma redução no número de aplicações de defensivos, garantindo uma estratégia ambiental e econômica mais amigável.

Portanto, o objetivo do trabalho foi caracterizar e espacializar por meio de sistemas de informações geográficas (SIG) o risco climático de ocorrência das doenças fúngicas (antracnose, míldio e manchas-dasfolhas), para a uva de mesa 'Niagara Rosada' na região do Polo Turístico do Circuito das Frutas, utilizando o modelo de previsão de época de pulverização, com base na ocorrência de chuva, utilizando séries históricas de dados de precipitação pluvial.

\section{MATERIAL E MÉTODOS}

A região de estudo é compreendida pelos municípios do Polo Turístico do Circuito das Frutas: Indaiatuba, Itupeva, Itatiba, Jarinu, Jundiaí, Louveira, Valinhos, Vinhedo, Morungaba e Atibaia (Figura 1). A área está localizada entre as coordenadas geográficas $22^{\circ} 50^{\prime}$ e $23^{\circ} 20^{\prime}$ de latitude Sul e $46^{\circ} 25^{\prime}$ e $47^{\circ} 18^{\prime}$ de longitude Oeste, com área aproximada $2.386 \mathrm{~km}^{2} \mathrm{e}$ cerca de um milhão de habitantes (IBGE, 2008). É uma região que abrange três tipos climáticos distintos, todos correspondentes a climas úmidos. A Região Oeste possui o clima tropical de altitude, classificado como Cwa, com inverno seco e verão quente. Já na área central, o clima é o subtropical, com verão quente, sem estação seca, sendo do tipo Cfa. A Região Leste do circuito, que incluem áreas serranas como a Serra do Japi é do tipo $\mathrm{Cfb}$, é caracterizada pelo verão ameno e chuvas o ano todo.

O risco climático $(R C)$ de ocorrência de doenças fúngicas na videira 'Niagara Rosada', definido como a relação entre o número de pulverizações necessárias devido à ocorrência de chuvas $\left(N P_{\text {chuva }}\right)$ e o número máximo de pulverizações possíveis baseadas no calendário, considerando-se que éfeita uma pulverização a cada sete dias $\left(N P_{\text {calendário }}\right)$ em função da duração do ciclo: poda-colheita, expresso em porcentagem, foi estimado conforme LOZADA GARCIA (2005):

$R C=\frac{N P_{\text {chuva }}}{N P_{\text {calendário }}} \times 100$ 


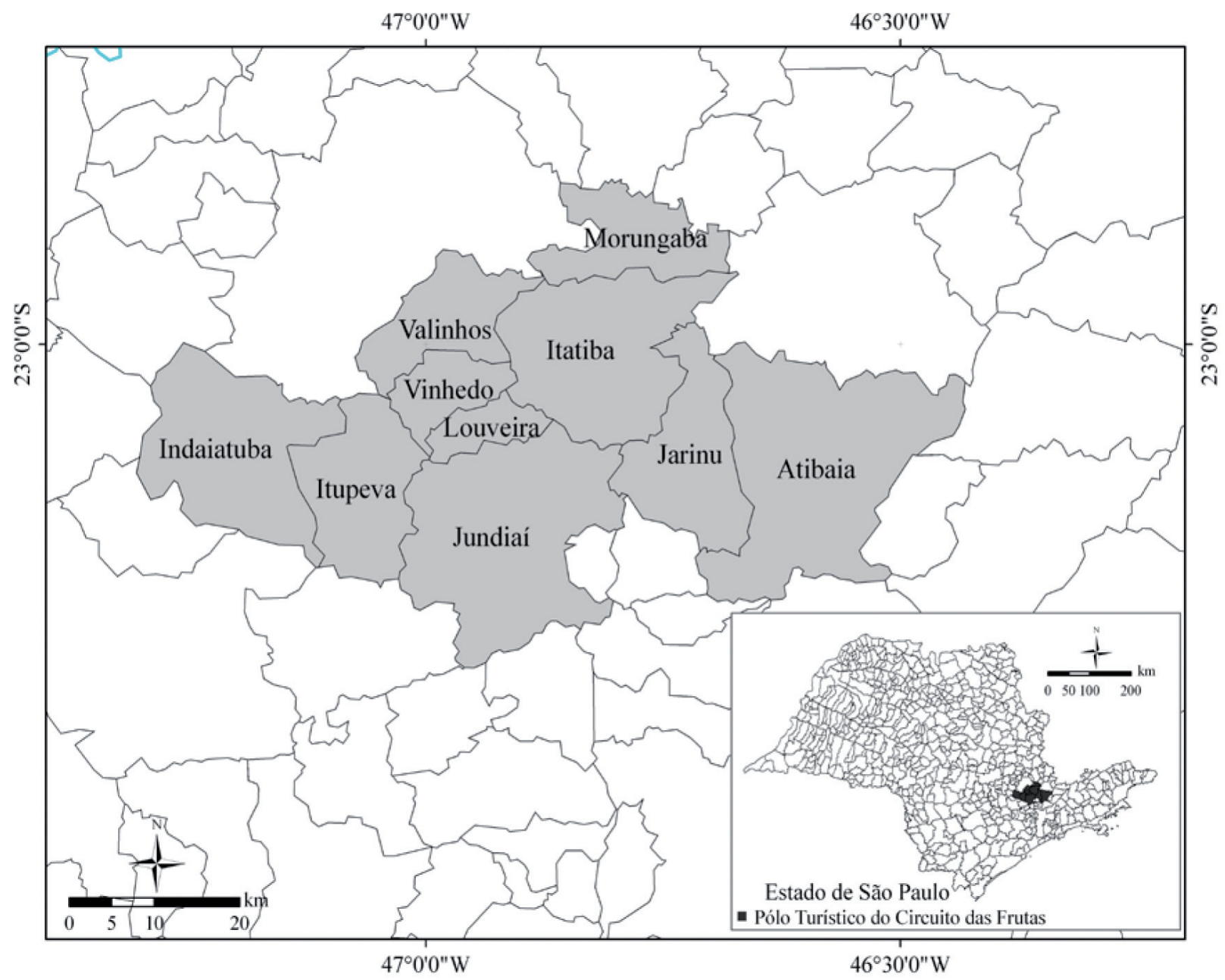

Figura 1. Municípios do Polo Turístico do Circuito das Frutas do Estado de São Paulo.

O $R C$ foi calculado para as diferentes épocas prováveis de colheita, referentes a cada época de poda, nos diferentes municípios da região. Foram utilizados valores diários de precipitação pluvial referentes a séries de 50 anos (1954-2003), pertencentes ao Departamento de Águas e Energia Elétrica (DAEE) e ao Instituto Agronômico (IAC), de Campinas, distribuídos nos municípios integrantes do Polo Turístico do Circuito das Frutas e em alguns municípios vizinhos.

O número de pulverizações necessárias para controle de doenças fúngicas na videira 'Niagara Rosada' foi determinado segundo PEDRO JúNIOR et al. (1999), utilizando o sistema pluviométrico para recomendação de época de aplicação de fungicidas. As aplicações são feitas após a ocorrência de períodos que totalizem $20 \mathrm{~mm}$ de chuva, respeitando a carência de sete dias do produto comercial. Para o cálculo do número de pulverizações necessárias $\left(N P_{\text {chuva }}\right)$, em função da ocorrência de chuvas foram assumidas seguintes premissas: a) os inóculos das doenças avaliadas (antracnose, míldio e mancha-dasfolhas) estão sempre presentes nas áreas de cultivo; b) a cultivar 'Niagara Rosada' é suscetível aos patógenos.

O número de pulverizações necessárias foi calculado em função da duração do ciclo (podacolheita) estimada para os diferentes locais, pelo acúmulo de graus-dia, até o total de 1549, considerando a temperatura-base de $10{ }^{\circ} \mathrm{C}$ (PEDRo JúnIOR et al., 1994). $\mathrm{O}$ acúmulo de chuva para indicação de pulverização se iniciou em $25 / 7$ (poda: $15 / 7$ ); $25 / 8$ (poda: $15 / 8$ ) e $25 / 9$ (poda 15/9), pois representa o tempo necessário para que ocorra a brotação, sendo a contagem finalizada dez dias antes da data estimada de colheita.

A distribuição espacial do $R C$ foi feita usando os valores médios do $R C$ que foram ajustados por equações de regressão linear simples em função da altitude:

$R C_{e s t}=a+b .($ alt $)$ 
sendo, $R C_{\text {est }}=$ valor do risco climático estimado; $a$ e $b=$ coeficientes da equação de regressão e alt $=$ altitude (metros). $\mathrm{O}$ teste $\mathrm{F}$ foi utilizado para avaliar a significância das equações de regressão e o coeficiente de determinação $\left(\mathrm{R}^{2}\right)$ ao nível de $99 \%$ de probabilidade.

Uma vez obtidas as equações de regressão procedeu-se a espacialização dos valores de $R C$, sendo realizada uma interpolação de dados com auxílio do sistema de informação geográfica (SIG) - ILWIS ${ }^{(5)}$. Foi utilizado o modelo digital de elevação do terreno (MDE), com resolução espacial de $90 \mathrm{~m}$, obtido por sensoriamento remoto orbital, (SRTM, 2006). A altitude da região varia de $520 \mathrm{~m}$ na região de Indaiatuba e se eleva à medida que se vai para leste, chegando a altitudes superiores a 1400 m em Atibaia e também, em Jundiaí, na Serra do Japi. Foram desenvolvidos mapas de risco climático para três diferentes épocas de poda (15/julho, 15/agosto e 15/ setembro), sendo as imagens reclassificadas, para melhor visualização dos valores de risco.

\section{RESULTADOS E DISCUSSÃO}

$\mathrm{O} N P_{\text {chuva }}$ foi estimado para as séries de 50 anos e nas tabelas 1, 2 e 3 são apresentados os valores médios e desvio-padrão, para cada época de poda e provável de colheita, nos municípios integrantes do Polo Turístico do Circuito das Frutas.

Tabela 1. Valores médios do número de pulverizações necessárias devido à ocorrência de chuvas ( $\left.N P_{\text {chuva }}\right)$, desvio-padrão e número máximo de pulverizações possíveis, baseadas no calendário ( $N P_{\text {calendário }}$, para podas efetuadas em 15 de julho, na região do Polo Turístico do Circuito das Frutas

\begin{tabular}{lcccc}
\hline & \multicolumn{4}{c}{$N P_{\text {chuva }}$} \\
\cline { 2 - 5 } Local & \multicolumn{4}{c}{ Data provável de colheita } \\
\cline { 2 - 5 } & $30 /$ nov. & $15 /$ dez. & $31 /$ dez. & \\
\hline Indaiatuba & $5,2 \pm 1,5$ & $6,4 \pm 1,6$ & $7,5 \pm 1,6$ & $8,5 \pm 1,7$ \\
Itupeva & - & $6,2 \pm 1,6$ & $7,3 \pm 1,6$ & $8,4 \pm 1,7$ \\
Valinhos & - & $6,6 \pm 1,6$ & $7,8 \pm 1,6$ & $9,0 \pm 1,7$ \\
Vinhedo & - & $6,5 \pm 1,7$ & $7,6 \pm 1,7$ & $8,6 \pm 1,8$ \\
Louveira & - & $6,6 \pm 1,5$ & $7,8 \pm 1,4$ & $8,9 \pm 1,4$ \\
Jundiaí & - & $6,4 \pm 1,5$ & $7,6 \pm 1,5$ & $8,7 \pm 1,5$ \\
Morungaba & - & $7,0 \pm 1,5$ & $8,2 \pm 1,6$ & $9,4 \pm 1,5$ \\
Jarinu & - & $7,1 \pm 1,5$ & $8,2 \pm 1,6$ & $9,4 \pm 1,6$ \\
Itatiba & - & $6,5 \pm 1,6$ & $7,6 \pm 1,8$ & $8,9 \pm 1,7$ \\
Atibaia & - & $6,7 \pm 1,7$ & $8,0 \pm 1,8$ & $9,1 \pm 1,7$ \\
NPcalendário: & 16 & 18 & 20 & 22 \\
\hline
\end{tabular}

-Não ocorre data provável de colheita na localidade.

Tabela 2. Valores médios do número de pulverizações necessárias devido à ocorrência de chuvas $\left(N P_{\text {chuva }}\right)$, desvio-padrão e número máximo de pulverizações possíveis, baseadas no calendário ( $\left.N P_{\text {calendário }}\right)$, para podas efetuadas em 15 de agosto, na região do Polo Turístico do Circuito das Frutas

\begin{tabular}{lcccc} 
Local & \multicolumn{4}{c}{ Data provável de colheita } \\
& $31 /$ dez. & $15 /$ jan. & $31 /$ jan. & $15 / \mathrm{fev.}$ \\
\hline Indaiatuba & $6,8 \pm 1,3$ & $7,9 \pm 1,4$ & $9,1 \pm 1,4$ & - \\
Itupeva & $6,5 \pm 1,3$ & $7,7 \pm 1,5$ & $9,0 \pm 1,5$ & $10,9 \pm 1,4$ \\
Valinhos & $7,2 \pm 1,3$ & $8,4 \pm 1,4$ & $9,8 \pm 1,5$ & $10,4 \pm 1,6$ \\
Vinhedo & $6,9 \pm 1,3$ & $8,0 \pm 1,4$ & $9,3 \pm 1,6$ & - \\
Louveira & $7,1 \pm 1,2$ & $8,3 \pm 1,2$ & $9,6 \pm 1,2$ & $10,4 \pm 1,4$ \\
Jundiaí & $6,9 \pm 1,2$ & $8,1 \pm 1,2$ & $9,4 \pm 1,4$ & $11,2 \pm 1,4$ \\
Morungaba & - & $8,7 \pm 1,3$ & $10,0 \pm 1,4$ & - \\
Jarinu & - & $8,7 \pm 1,3$ & $9,7 \pm 1,5$ & $10,6 \pm 1,7$ \\
Itatiba & - & $8,3 \pm 1,6$ & $9,6 \pm 1,7$ & $10,0 \pm 1,6$ \\
Atibaia & - & $8,6 \pm 1,5$ & 20 & 22 \\
NPcalendário: & 16 & 18 & & $20,9 \pm 1,6$ \\
\hline
\end{tabular}

- Não ocorre data provável de colheita na localidade. 
Tabela 3. Valores médios do número de pulverizações necessárias devido à ocorrência de chuvas $\left(N P_{\text {chuva }}\right)$, desvio-padrão e o número máximo de pulverizações possíveis baseadas no calendário ( $N P_{\text {calendário }}$, para podas efetuadas em 15 de setembro, na região do Polo Turístico do Circuito das Frutas

\begin{tabular}{|c|c|c|c|c|}
\hline \multirow{3}{*}{ Local } & \multicolumn{4}{|c|}{$N P_{\text {chuva }}$} \\
\hline & \multicolumn{4}{|c|}{ Data provável de colheita } \\
\hline & 25/jan. & 10/fev. & 25/fev. & 10/mar. \\
\hline Indaiatuba & $7,7 \pm 1,3$ & $8,8 \pm 1,4$ & - & - \\
\hline Itupeva & $7,6 \pm 1,3$ & $8,9 \pm 1,3$ & - & - \\
\hline Valinhos & $8,2 \pm 1,3$ & $9,5 \pm 1,3$ & $10,6 \pm 1,5$ & - \\
\hline Vinhedo & $7,8 \pm 1,4$ & $9,1 \pm 1,6$ & $10,2 \pm 1,6$ & - \\
\hline Louveira & $8,0 \pm 1,2$ & $9,3 \pm 1,3$ & - & - \\
\hline Jundiaí & $7,9 \pm 1,2$ & $9,1 \pm 1,3$ & $10,2 \pm 1,3$ & $11,1 \pm 1,4$ \\
\hline Morungaba & $8,6 \pm 1,1$ & $10,6 \pm 1,3$ & $11,0 \pm 1,2$ & $12,1 \pm 1,4$ \\
\hline Jarinu & $8,3 \pm 1,3$ & $9,5 \pm 1,4$ & $10,7 \pm 1,5$ & - \\
\hline Itatiba & $7,9 \pm 1,3$ & $9,3 \pm 1,5$ & $10,4 \pm 1,5$ & $11,5 \pm 1,6$ \\
\hline Atibaia & $8,2 \pm 1,4$ & $9,6 \pm 1,5$ & $10,8 \pm 1,5$ & $12,2 \pm 1,7$ \\
\hline NPcalendário: & 15 & 17 & 19 & 21 \\
\hline
\end{tabular}

- Não ocorre data provável de colheita na localidade.

Devido à influência da temperatura na duração do ciclo da videira, observou-se que em alguns municípios com temperaturas mais baixas, prolongando a duração do ciclo, não houve data provável de colheita anterior a 30 de novembro para podas efetuadas em $15 /$ julho (tabela 1). O mesmo fato ocorreu para podas efetuadas em 15 de agosto em relação à data provável de colheita de 31 de dezembro (Tabela 2). Por outro lado, em municípios com temperatura mais elevada, onde o ciclo da videira é mais curto, não houve datas prováveis de colheita em 15 de fevereiro (Tabela 2) e 25 de fevereiro e 10 de março (Tabela 3).

Pode-se observar que nos municípios de Itupeva e Indaiatuba os valores de $N P_{\text {chuva }}$ foram baixos, sendo esses locais situados em altitudes menos elevadas, com maiores temperaturas e menores índices de chuva. Por outro lado os maiores valores de $N P_{\text {chuva }}$ ocorreram, principalmente, nos municípios de Morungaba, Atibaia, Itatiba e Jarinu. Esses locais são considerados mais chuvosos e com temperaturas mais amenas, proporcionando um ciclo mais longo da videira, sendo necessário maior número de pulverizações.

Para um mesmo município e mesma época de poda, podem ocorrer diferentes épocas de colheita, em função da variação espacial da temperatura do ar existente. Assim, algumas regiões atingem o total de graus-dias necessários para a maturação da uva mais precocemente. No município de Indaiatuba, é possível observar que há, em média, o aumento de uma pulverização, com a prolongação do ciclo em 15 dias, fato também observado por PEDRO JúnIOR et al. (1999) na região de Jundiaí. Já em locais com maior índice pluviométrico, o prolongamento de 15 dias no ciclo da 'Niagara Rosada', indicam a necessidade de duas pulverizações a mais, como observado para os municípios de Morungaba, Atibaia e Itatiba.

Os valores médios de $N P_{\text {chuva }}$ mais elevados foram observados para a poda de 15 de setembro, devido ao período de crescimento e à produção da videira ocorrer durante as estações da primavera e verão, meses considerados mais chuvosos. O menor número observado de pulverizações foi igual a oito, uma a mais se comparado com a poda de 15 de agosto e até três pulverizações a mais, se comparado com o menor valor necessário para a poda efetuada em 15 de julho.

Para a poda de 15 de julho, foi necessário um menor número de pulverizações em comparação com as podas de 15 de agosto e 15 de setembro, devido ao fato do crescimento das plantas ocorrerem em período menos chuvoso. Resultados semelhantes foram analisados na região de Jundiaí por PEDRO JúNIOR et al. (1999), onde em geral o número de pulverizações utilizadas para o controle de doenças fúngicas são menores para a poda efetuadas em julho. Os autores observaram que para o ano agrícola 1996/1997, o número de pulverizações aumentam quando a poda é mais tardia. Em outros anos, observou-se que a poda efetuada em agosto necessitou de maior número de pulverizações, se comparada com a de julho e setembro.

O $N P_{\text {chuva }}$ foi utilizado para estimativa do risco climático $(R C)$ e para a espacialização dos valores de $R C$ foram desenvolvidas equações de regressão para sua estimativa em função da altitude. Na tabela 4, são apresentados os coeficientes das equações de regressão simples, para estimativa do risco climático em função da altitude, obtidas para cada época de poda, para o Polo Turístico do Circuito das Frutas. Também são 

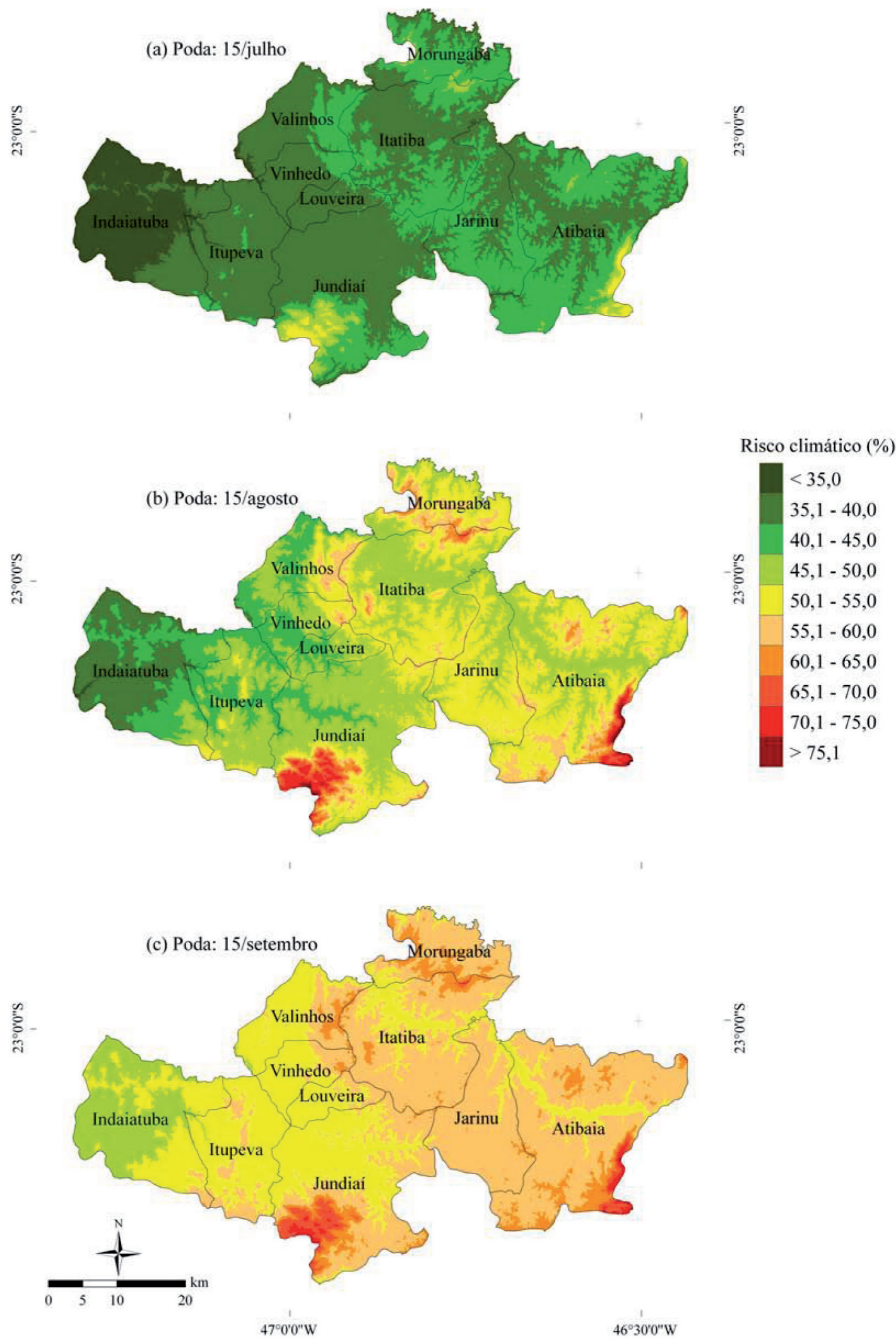

Figura 2. Risco climático de ocorrência de doenças fúngicas na videira 'Niagara Rosada' na região do Polo Turístico do Circuito das Frutas, para as podas efetuadas em: (a) 15 de julho, (b) 15 de agosto e (c) 15 de setembro. 
Tabela 4. Coeficientes dos modelos de regressão simples para estimativa o índice de risco climático e coeficientes de determinação $\left(R^{2}\right)$ para diferentes épocas de poda da videira 'Niagara Rosada' no Polo Turístico do Circuito das Frutas

\begin{tabular}{lccc}
\hline Data de poda & Constante & Coeficiente angular da altitude & $\mathrm{R}^{2}$ \\
\hline 15 de julho & 15,91 & $0,02973^{* *}$ & $0,68^{* *}$ \\
15 de agosto & 2,71 & $0,05958^{* *}$ & $0,70^{* *}$ \\
15 de setembro & 27,35 & $0,03623^{* *}$ & $0,58^{* *}$ \\
\hline
\end{tabular}

** = significativo ao nível de $1 \%$.

apresentadas as significâncias dos estimadores das equações.

Os valores dos coeficientes de determinação $\left(R^{2}\right)$ obtidos na estimativa do risco climático variaram entre 0,58 (poda em setembro) e 0,70 (poda em agosto) tendo sido estatisticamente significativos ao nível de $1 \%$ de probabilidade.

A seguir, são apresentados os mapas de risco climático de ocorrência de doenças fúngicas na videira 'Niagara Rosada', para a região do Polo Turístico do Circuito das Frutas (Figura 2), considerando-se as seguintes épocas de poda: 15 /julho, 15 /agosto e $15 /$ setembro.

Para a poda em 15 de julho, observa-se que no município de Indaiatuba, o $R C$ é inferior a $35 \%$, e esse valor aumenta em direção à Região Leste da área estudada. Em cerca de $42 \%$ da área, o risco está entre $35 \%$ e $40 \%$. Os maiores valores de risco podem ser observados em áreas mais elevadas nos municípios de Jundiaí e Atibaia, com valores que atingem $55 \%$.

O RC para a poda de 15 de agosto representa um aumento nos valores em relação à poda de $15 /$ julho. A região de Indaiatuba continuou com valores mais baixos, entre $35 \%$ e $50 \%$. Para a maior parte da região $(30 \%)$ os valores de $R C$ atingiram $55 \%$, porém observa-se que, em algumas áreas localizadas em Morungaba, Jundiaí e Atibaia, o risco atinge valores próximos a $75 \%$.

Para a poda de 15 de setembro, observa-se que os valores de $R C$ aumentam em relação às podas anteriores, porém em algumas áreas há valores entre $45 \%$ e $55 \%$. Na faixa que abrange a Região Central e Leste do Circuito das Frutas, representando 50\% da região, o $R C$ varia entre $55 \%$ e $60 \%$. Os valores de $R C$ mais elevados se concentram em pequenas áreas, nos municípios de Morungaba, Jundiaí e Atibaia, assim como observado para a poda de 15 de agosto, como valores próximos a $70 \%$.

Considerando as diferentes épocas de poda, observa-se que o $R C$ aumenta quando a poda é mais tardia em função do aumento da pluviosidade. Em 15 de setembro os valores do $R C$ variaram entre $45 \%$ e $75 \%$. Para as outras datas de poda: 15 de julho e 15 de agosto, os valores de risco, variaram entre $<35 \%$ e
$55 \%$, e 35 e $75 \%$ respectivamente. De maneira geral, pode-se observar que os valores de risco aumentam da Região Oeste para Leste, e em direção a regiões mais elevadas, independentemente da época de poda. Essa variação acompanha o aumento de duração do ciclo da 'Niagara Rosada' em áreas mais frias e, também, as regiões mais chuvosas, correspondentes à área de maior altitude.

A variação observada do $R C$ pode servir de indicação para os viticultores na escolha de áreas com menor risco para implantação de vinhedos, evitando áreas onde os valores de $R C$ são altos, e demandam maior número de pulverizações no controle de doenças fúngicas. $\mathrm{O}$ viticultor também poderá antecipar ou retardar a poda da videira, em função do risco, alterando o número de pulverizações necessárias, gerando uma economia no gasto de defensivos agrícolas e aumentando a preservação ambiental.

Além disso, a geração de mapas facilita a visualização da distribuição das áreas de risco ou de escape à ocorrência de doenças, as quais poderão contribuir no planejamento do sistema produtivo ou na expansão das áreas produtoras de uva na região do Polo Turístico do Circuito das Frutas.

\section{CONCLUSÕES}

1. Os valores de risco climático estão associados à época de poda. Nas podas efetuadas em setembro os riscos são mais elevados que nas de julho.

2. Na Região Oeste do Polo Turístico do Circuito das Frutas, o risco climático de ocorrência de doenças fúngicas na 'Niagara Rosada' é inferior quando comparada com a da Região Leste.

3. Em áreas de maior altitude, o risco climático de ocorrência de doenças é mais elevado, sendo possível sua estimativa por meio de equações de regressão em função da altitude.

\section{AGRADECIMENTOS}

À Fundação de Amparo à Pesquisa do Estado de São Paulo (FAPESP) pela concessão da bolsa de Pósgraduação (Mestrado) para Ludmila Bardin. 


\section{REFERÊNCIAS}

BLEICHER, J.O. O sistema pluviométrico para previsão da podridão amarga da macieira. Agropecuária Catarinense, v.4, p.16-18, 1991.

BOOTH, T.H.; JOVANOULO, T.; OLD, K.M.; DUDZINSKI, M.L. Climatic mapping to identify high-risk areas in mainland south East Asia and around the word. Environmental Pollution, v.108, p.365-372, 2000.

IBGE-Instituto Brasileiro de Geografia e Estatística. Disponível em: http:/ / www.ibge.gov.br/ cidadesat/topwindow.htm?1. Acesso em dezembro de 2008.

LOZADA GARCÍA, B. I. Risco climático de ocorrência da requeima da batata na região dos Andes, Venezuela. 2005. 132p. Tese (Doutorado em Agronomia) - Escola Superior de Agricultura "Luiz de Queiroz", Universidade de São Paulo, Piracicaba.

PEDRO JÚNIOR M.J.; SENTELHAS P.C.; POMMER, C.V. Determinação da temperatura-base, graus-dia e índice biometeorológico para a videira 'Niagara Rosada'. Revista Brasileira de Agrometeorologia, v.2, p.51-56, 1994.
PEDRO JÚNIOR, M.J.; PEZZOPANE, J.R.M.; MARTINS, F.P. Uso da precipitação pluvial para previsão de épocas de pulverização visando controle de doenças fúngicas na videira 'Niagara Rosada'. Revista Brasileira de Agrometeorologia, v.7, p.107-111, 1999.

PEZZOPANE, J.R.M., PEDRO JÚNIOR., M.J, MORAES, S.A., GODOY, I.J., PEREIRA, J.C.V.N.A.; SILVEIRA, L.C.P. Chuva e previsão de épocas de pulverização para controle das manchas foliares do amendoim. Bragantia, v.57, p.285295, 1998.

SÔNEGO, O. R.; GARRIDO, L. R. Capacitação Técnica em Viticultura. Empresa Brasileira de Pesquisa Agropecuária EMBRAPA. Disponível em: http:/ / www.cnpuv.embrapa.br / viticultura. Acesso em agosto de 2006.

SRTM - Shuttle Radar Topography Mission. Disponível em: http: / / srtm.usgs.gov. Acesso em agosto de 2006.

ZEUNER, T.; KLEINHENZ, B. Use of geographic information systems in warning services for late blight. Bulletin OEPP/ EPPO, v.37, p.327-334, 2007. 